\title{
Biología del grupo prehistórico de Coyo Oriental (San Pedro de Atacama, norte de Chile): II Deformación craneana artificial
}

José A. Cocilovo ${ }^{1}$ Y María V. ZaVATtieri ${ }^{1}$

\begin{abstract}
RESUMEN
La deformación artificial del cráneo constituye un importante factor de variación intrapoblacional que, además del interés intrínseco que despierta su estudio, representa una fuente de error considerable cuando se estima el parentesco entre dos o más grupos. En el presente trabajo se realiza el análisis del influjo de los tipos tabular erecto, circular erecto y oblicuo en la morfogénesis craneana de los materiales exhumados en el sitio Coyo Oriente de San Pedro de Atacama. Se dispuso de una muestra de 158 cráneos de ambos sexos y de edades adulto, maduro y senil, a partir de los cuales se obtuvo información sobre 53 variables métricas. Los datos fueron transformados para evitar el efecto del sexo y de la edad, procedimiento que permitió una evaluación más objetiva de las diferencias producidas por las distintas prácticas culturales. Se controlaron los supuestos de distribución normal y el de homogeneidad entre varianzas. Las diferencias entre medias fueron docimadas por medio de un análisis de la varianza de una vía. Los resultados obtenidos reflejan importantes diferencias entre los valores medios de ejemplares deformados y normales, a nivel de las dimensiones generales de la bóveda craneal, y de las anchuras de la base del cráneo, comprobándose además efectos menores en el esplacnocráneo. Estas observaciones son comparadas con la información obtenida en otras experiencias realizadas con materiales del norte de Chile, Noroeste Argentino y Patagonia.
\end{abstract}

\section{Introducción}

La deformación artificial de la cabeza constituye una conspicua práctica cultural que modifica sensiblemente la forma del cráneo, representando por ello un importante factor de variación intrapoblacional. La preocupación por este tema en la literatura científica se pone de manifiesto en los trabajos de Imbelloni (1925, 1932, 1933, 1950), quien propone varios tipos y formas y analiza su distribución geográfica, en la obra de Dembo e Imbelloni (s/f) o en los clásicos estudios de Hrdliçka (1910), Oettking (1922), Falkenburger (1938), Neuman (1942) y Weiss $(1961,1962)$.

1 Departamento de Ciencias Naturales, Facultad de Ciencias Exactas, Físico-Químicas y Naturales, Universidad Nacional de Río Cuarto, 5800 Río Cuarto, ARGENTINA.
En 1958 se realiza una experiencia destinada a comprender las alteraciones sufridas por la base craneal debidas al efecto de la deformación artificial (Moss 1958). Posteriormente, aparece un trabajo más completo que proporciona información sobre las modificaciones sufridas a nivel de varias estructuras craneales (McNeill y Newton 1965). En estos ensayos se observan discrepancias en las conclusiones que seguramente dependen de la calidad del diseño experimental aplicado en cada caso (Cocilovo 1978). Ossemberg (1970) realiza una serie de observaciones sobre el influjo de esta práctica en la expresión de los rasgos discretos, las que permiten inferir un menor crecimiento y desarrollo óseo en el frontal y en la bóveda posterior, opuestos a la expansión de la bóveda lateral y de la base craneal. El influjo de la deformación artificial es analizado por Pucciarelli en cráneos indígenas $\mathrm{y}$ en forma experimental en ratas de laboratorio (Pucciarelli 1972, 1973a, 1973b, 1974).

El efecto de la deformación tabular erecta fue estudiado estadísticamente en un grupo de Patagonia (Cocilovo 1973, 1978), a partir de lo cual se obtiene un conocimiento mayor de los cambios producidos en la morfogénesis craneana. En 1975 se intenta un ensayo similar, con escasos resultados, en materiales de Paucarcancha (Perú) deformados circularmente (Cocilovo 1975). La imagen más clara del influjo de la deformación circular, en sus formas erecta y oblicua, es obtenida por una serie de trabajos realizados en la colección osteológica de Morro de Arica (Mendonça y Di Rienzo1981-82; Cocilovo et al. 1982; Mendonça et al. 1983, 1986; Di Rienzo y Cocilovo 1984). Posteriormente, se analiza en forma global la incidencia de varios tipos deformatorios en Pisagua (Cocilovo, en prensa) y en San Pedro de Atacama (Cocilovo y Varela Ms), en La Paya (Cocilovo y Baffi 1985) y en Las Pirguas (Baffi Ms). Los efectos de una particular práctica definida como "pseudocircular" son analizados en los materiales de Laguna del Juncal, trayendo como consecuencia su asignación al tipo circular (Guichón Ms). 
A partir de los citados trabajos, disponemos hoy de una información mucho más detallada acerca de los reales efectos de la deformación artificial del cráneo humano basada en diseños experimentales más apropiados y en el control de una serie de supuestos subyacentes que tradicionalmente fueron ignorados.

Dentro de estos supuestos se incluye, por ejemplo, la ausencia de variabilidad geográfica y cronológica, de dimorfismo sexual y de variabilidad etaria, además de aquellos vinculados con la distribución de las variables métricas. Los distintos tipos y formas de prácticas culturales aplicados con la finalidad de modificar la morfología del cráneo humano constituyen un poderoso factor de variación a nivel local y regional. Por ello, además de su interés intrínseco, el estudio de la deformación como factor de variabilidad permite determinar su incidencia en la comparación de grupos, a fin de elaborar estrategias alternativas y procedimientos de análisis más objetivos en la estimación de distancias biológicas (Varela et al. en prensa).

Coyo Oriente es un sitio ubicado a $5 \mathrm{~km}$ al sursureste de San Pedro de Atacama en el norte de Chile (Figura 1), excavado por el R. P. Le Paige en la década de 1950. El análisis de los materiales culturales (Berenguer y Dauelsberg 1989) reflejó un incremento de la presencia altiplánica en el área, entre 600 y 1000 DC, a través de Tiwanaku. Estos materiales están asociados a una colección de restos óseos, lo que permite disponer de un importante marcador biológico para inferir la naturaleza y la significación de las interacciones biosociales locales y regionales. Para cumplir con

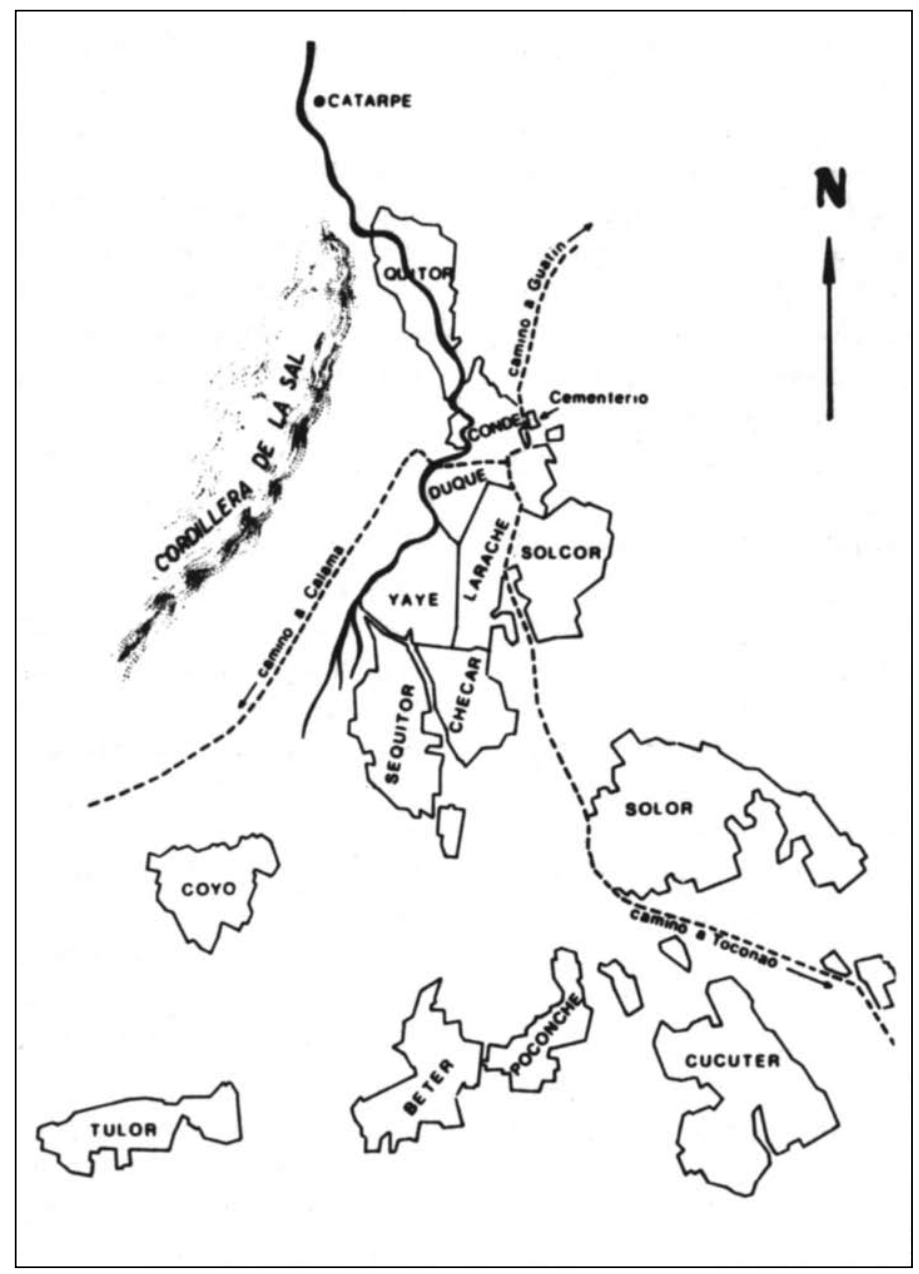

Figura 1. Distribución de los ayllu de San Pedro de Atacama. Redibujado a partir de Le Paige (1963), reducción al 90\% del original (Esc. 1:50000). 
este objetivo es necesario analizar previamente la manifestación del dimorfismo sexual, de la variación etaria y de la deformación artificial. Así, en un trabajo anterior nos ocupamos de los dos primeros factores (Cocilovo et al. 1996), mientras que en el presente lo hacemos con referencia a la deformación artificial existente en la mayoría de los ejemplares exhumados en el citado cementerio. El hecho de que parte de las piezas de este grupo presenten un desarrollo normal, como término objetivo de comparación, nos brinda naturalmente una excelente oportunidad para analizar la acción de las distintas prácticas culturales.

Munizaga (1969), analizando materiales de distintas fases culturales de San Pedro de Atacama, comprueba un predominio de tabulares erectos en el momento inicial del Período Agroalfarero (Fase I de Le Paige), luego se tornan más frecuentes los tabulares oblicuos asociados con la cerámica Negra Pulida (Fase II de Le Paige). En la etapa siguiente vuelven a ser más frecuentes los primeros asociados con la cerámica Negra Casi Pulida (Fase III de Le Paige) y al final se observa un neto incremento de los tabulares erectos junto con las formas oblicuas. Costa (1985) informa para Quitor 6 (sector tardío 940-1240 DC) la existencia de varios tipos de prácticas culturales definidas como "frontal", "parietal", "lamboidal" y "frontooccipital". Por las descripciones dadas, los tres primeros tipos pueden ser adscritos, según el sistema de Imbelloni (1925), como tabulares erectos y tabular oblicuo el último, lo que a su vez coincidiría con las citadas observaciones de Munizaga.

\section{Material y métodos}

La presente experiencia fue realizada con los materiales de la colección del sitio Coyo Oriente, constituida por 158 ejemplares de ambos sexos y de edades adulto, maduro y senil (Tabla 1). Durante el relevamiento se comprobó que un conjunto de piezas no presentaba deformación artificial, mientras que en mayor proporción se observó la práctica de distintos tipos deformatorios: tabular erecto, circular erecto y oblicuo, siendo estos últimos menos frecuentes.

Se relevaron 53 variables métricas según la Convención Internacional de Mónaco (en Comas 1966) y Wilder (1920). El diagnóstico de edad y sexo se realizó de acuerdo con las síntesis de Bordach (1985 y 1989) y el de deformación artificial por Imbelloni (1925 y 1933).

\begin{tabular}{|l|c|c|c|c|c|c|c|}
\hline \multirow{2}{*}{$\begin{array}{c}\text { Edadlsexo } \\
\text { deformación }\end{array}$} & \multicolumn{2}{|c|}{ Adulto } & \multicolumn{2}{c|}{ Maduro } & \multicolumn{2}{c|}{ Senil } & \multirow{2}{*}{ Total } \\
\cline { 2 - 9 } & M & F & M & F & M & F & \\
\hline Tab. erecta & 7 & 15 & 24 & 20 & 4 & 11 & 81 \\
\hline Circ. erecta & 1 & 4 & 1 & 1 & & & 7 \\
\hline Circ. oblicua & 1 & & & 2 & & 1 & 4 \\
\hline No deformados & 3 & 11 & 20 & 21 & 6 & 5 & 66 \\
\hline \multicolumn{1}{|c|}{ Total } & 42 & \multicolumn{3}{|c|}{89} & \multicolumn{2}{|c|}{27} & 158 \\
\hline
\end{tabular}

Tabla 1. Composición y estructura de la colección de Coyo Oriental.

Con el objeto de analizar la variación provocada por la deformación artificial en la morfogénesis craneana, se confrontaron los valores medios de cada uno de los grupos deformados y normales mediante una técnica de análisis de la varianza de una vía. Previamente, se controlaron los supuestos de distribución normal (Rao 1952; David et al. 1954; Shapiro y Wilk 1965) y de homogeneidad entre varianzas (Bliss 1967). En nuestro caso la prueba de homocedasticidad se realizó mediante el estadístico M', que se distribuye como una $\mathrm{Chi}^{2}$ con 3 grados de libertad, y el cociente entre varianzas permitió probar la igualdad entre medias, distribuyéndose como una $\mathrm{F}$ con 3 y $\mathrm{n}$ grados. Como en experiencias anteriores con estos materiales (Cocilovo et al. Ms), hemos trabajado con los datos libres de la variación producida por el dimorfismo sexual y la edad, mediante una transformación de los mismos que elimina el influjo de dichos factores.

\section{Resultados y discusión}

Los resultados de la presente experiencia se exponen en las Tablas 2 y 3 . En la primera se consigna, para cada variable y para cada grupo, el número de observaciones (N), la media (MD), la desviación estándar (S) y las pruebas de distribución (asimetría y curtosis). En la segunda figuran las pruebas de homogeneidad entre varianzas (M') y de diferencia entre valores medios (F). Para esta última se especifican los grados de libertad del denominador (gl). Hay un conjunto de variables para las cuales no se dispuso del número mínimo de observaciones necesario para realizar los cálculos, por lo que figuran sin datos en las tablas.

El 51\% de la muestra estudiada presenta una deformación de tipo tabular erecta, $7 \%$ circular y $42 \%$ no presenta señales de deformación. Por otra parte, según se observa en la Tabla 1, no parece existir asociación con el sexo ni con la edad. 


\begin{tabular}{|c|c|c|c|c|c|c|c|c|c|c|c|c|}
\hline \multirow{2}{*}{ Variables } & \multicolumn{3}{|c|}{ Tabulares erectos } & \multicolumn{3}{|c|}{ Circulares erectos } & \multicolumn{3}{|c|}{ Circulares oblicuos } & \multicolumn{3}{|c|}{ No deformados } \\
\hline & $\mathbf{N}$ & MD & $\mathbf{S}$ & $\mathbf{N}$ & MD & $\mathbf{S}$ & $\mathbf{N}$ & MD & $\mathbf{S}$ & $\mathbf{N}$ & MD & $\mathbf{S}$ \\
\hline Longitud máxima (glabela) & 80 & $126.836 \mathrm{a}$ & 6.249 & 7 & 165.337 & 5.947 & 4 & 172.517 & 4.584 & 66 & 170.932 & 4.296 \\
\hline Ancho máximo & 81 & 146.153 & 5.17 & 7 & 137.851 & 4928 & 4 & 136.745 & 6.261 & 66 & $141.017 \mathrm{ad}$ & 4.854 \\
\hline Altura basilobregmática & 80 & 13.763 & 4.277 & 7 & 135.407 & 3.485 & 4 & 131.938 & 3.931 & 65 & 131.113 & 3.602 \\
\hline Altura priobregmática & 80 & 114.732 & 3.689 & 7 & 117.784 & 3.291 & 4 & 113327 & 2.864 & 65 & 113.820 & 3.702 \\
\hline Diámetro frontal mínimo & 81 & 88.069 & 4.429 & 7 & 88.869 & 4.533 & 4 & 81.265 & 1.948 & 66 & $89.502 \mathrm{a}$ & 3.271 \\
\hline Diámetro frontal máximo & 81 & $116.232 \mathrm{~d}$ & 5.061 & 7 & 111.903 & 2.495 & 4 & 104.210 & 3.971 & 65 & 113.505 & 4.549 \\
\hline Ancho bimastoideo & 81 & 106.411 & 4.344 & 7 & $1 \mathrm{M} .843$ & 2.209 & 4 & 101.085 & 4.542 & 66 & 104.732 & 4.063 \\
\hline Ancho bimastoideo máximo & 81 & 126.109 & 4.030 & 7 & 123.116 & 3.410 & 4 & 118.750 & 2.403 & 66 & 123.459 & 4.522 \\
\hline Ancho biauricular & 81 & 106.727 & 3.739 & 7 & 103.361 & 2.249 & 4 & 104.675 & 2.856 & 66 & 104.862 & 4.269 \\
\hline Diámetro mastobasilar & 80 & 92.030 & 3.001 & 6 & 93.372 & 3.945 & 4 & 92.182 & 5.243 & 65 & 92.803 & 3.193 \\
\hline Diámetro alveolobasilar & 79 & $89.444 \mathrm{a}$ & 4.164 & 7 & 93.029 & 4.873 & 4 & 89.795 & 6.185 & 85 & 88.944 & 5.350 \\
\hline Longitud forámen magnum & 77 & 35.696 & 2.114 & 7 & 36.109 & 2.293 & 4 & 36.957 & 2.433 & 65 & 36.306 & 1.842 \\
\hline Ancho forámen magnum & 77 & 31.425 & 1.899 & 7 & 32.744 & 1.855 & 4 & 31.033 & 1.549 & 66 & 32.088 & 2.371 \\
\hline Ancho frontromalar & 81 & 101.265 & 3.108 & 7 & 100.351 & 4.960 & 4 & 97.693 & 3.511 & 66 & 101.504 & 3.236 \\
\hline Ancho biastérica & 81 & 110.201 & 4.916 & 7 & 106.633 & 4953 & 4 & 102.728 & 3.114 & 66 & 109.502 & 5.108 \\
\hline Diámetro nasion-sphenobasion & 81 & 69.535 & 2.539 & 7 & 70.353 & 3.777 & 4 & 68.217 & 3.317 & 66 & 69.730 & 2.630 \\
\hline Diámetro alvéolo-sphenobasion & 80 & $75.880 \mathrm{c}$ & 3.956 & 7 & $78.494 \mathrm{c}$ & 3.673 & 4 & 75.635 & 4.746 & 66 & 74.930 & 5.040 \\
\hline Diámetro basion-gnation & 10 & 106.099 & 5.089 & & & & & & & 6 & 106.500 & 8.121 \\
\hline Curva transversal & 80 & 321.991 & 10.945 & 7 & 319.617 & 7.345 & 4 & 310.827 & 12.224 & 66 & 315.889 & 10.112 \\
\hline Curva horizontal por opistocráneo. & 80 & 492.613 & 10.822 & 7 & 489.096 & 12.904 & 4 & 484.530 & 12.483 & 66 & 500.632 & 11.276 \\
\hline Curva nasion-bregma & 81 & $119.329 \mathrm{~b}$ & 5.479 & 7 & 122.484 & 4.593 & 4 & $121.680 \mathrm{~d}$ & 0.825 & 66 & 122.009 & 5.443 \\
\hline Curva bregma-lambda & 81 & $114.807 \mathrm{c}$ & 7.399 & 7 & 118.089 & 11.701 & 4 & 115.140 & 7.340 & 66 & 114.969 & 8.518 \\
\hline Curva lambda-opistion & 78 & $109.185 \mathrm{c}$ & 8.456 & 7 & $112.884 \mathrm{~d}$ & 11.189 & 4 & 120.820 & 7.985 & 66 & $118.245 \mathrm{~b}$ & 8.624 \\
\hline Ancho bicigomático & 78 & 132.014 & 3.363 & 7 & 131.870 & 4.273 & 4 & 130.250 & 4.410 & 6 & 131.404 & 3.773 \\
\hline Altura del pómulo & 81 & 22.023 & 1.990 & 7 & 20.811 & 1.760 & 4 & 23.245 & 1.226 & 65 & 21.668 & 2.426 \\
\hline Altura nasoalveolar & 80 & $67.909 \mathrm{bc}$ & 4.595 & 7 & 70.977 & 2.660 & 4 & 67.760 & 4.626 & 66 & 66.824 & 4.706 \\
\hline Altura de la nariz & 80 & 50.027 & 2.488 & 7 & $50.467 \mathrm{~d}$ & 1.388 & 4 & 49.613 & 2.878 & 66 & 49.643 & 2.402 \\
\hline Ancho apertura pyriformis & 80 & 25.321 & 1.586 & 7 & 24.321 & 1.130 & 4 & 24.440 & 1.527 & 66 & 24.879 & 1.731 \\
\hline Distancia interorbitaria anterior & 81 & 23.003 & 1.619 & 7 & 23.196 & 1.899 & 4 & $20.640 \mathrm{~b}$ & 1.007 & 66 & 22.577 & 2.030 \\
\hline Ancho biorbitario & 80 & 95.791 & 2.747 & 7 & 96.280 & 3.675 & 4 & $91.620 \mathrm{c}$ & 4.093 & 66 & 95.892 & 2.893 \\
\hline Ancho de la órbita & 81 & $37.785 \mathrm{c}$ & 1.739 & 7 & 38.130 & 1.634 & 4 & 36.950 & 2.348 & 66 & 38550 & 1.421 \\
\hline Altura de la órbita & 81 & 35.230 & 1.748 & 7 & 35.774 & 1.914 & 4 & 35.430 & 2.109 & 66 & 35505 & 1.824 \\
\hline Longitud del paladar & 80 & 41.863 & 2.492 & 7 & $41.401 \mathrm{~d}$ & 2.690 & 4 & 43.160 & 1.985 & 64 & 42.069 & 2.979 \\
\hline Ancho del paladar & 80 & 36.812 & 2.478 & 7 & 34.303 & 3.146 & 4 & 39.445 & 1.744 & 66 & 36.769 & 2.627 \\
\hline Altura órbita-alveolar & 17 & 39.937 & 3.430 & 7 & 40.271 & 2.365 & 4 & 40.998 & 2.341 & 61 & 39.688 & 4.109 \\
\hline Ancho bimaxilar máximo & 79 & 96.115 & 3.870 & 7 & 96.823 & 2.873 & 4 & 96.710 & 4.873 & 66 & 96.052 & 4.248 \\
\hline Altura nasion-gnation & 10 & 116.788 & 7.017 & & & & & & & 6 & 117.190 & 4.947 \\
\hline Ancho maxiloalveolar & 76 & 57.830 & 4.044 & 7 & 58.099 & 2.899 & 4 & 59.198 & 3.961 & 61 & 56.597 & 4.730 \\
\hline Longitud maxiloalveolar & 77 & 49.579 & 2.881 & 7 & 50.767 & 2.951 & 4 & 49.200 & 3.486 & 62 & 49.472 & 3.420 \\
\hline Ancho bicondíleo & 27 & 116.278 & 4.242 & & & & & & & 28 & 116.909 & 4.093 \\
\hline Ancho bigoníaco & 31 & 88.883 & 4.828 & & & & & & & 28 & 90.988 & 5.285 \\
\hline Longitud de la mandíbula & 31 & 76.858 & 3.762 & & & & & & & 27 & 76.537 & 5.742 \\
\hline Altura rama montante & 31 & 57.682 & 4.990 & & & & & & & 27 & 58.293 & 4.921 \\
\hline Altura rama montante mínima & 30 & $47.462 \mathrm{c}$ & 4.078 & & & & & & & 28 & $47.648 \mathrm{c}$ & 3.861 \\
\hline Ancho rama montante & 32 & 39.424 & 3.148 & & & & & & & 27 & 38.938 & 3.407 \\
\hline Ancho rama montante mínimo & 32 & 30.142 & 2.887 & & & & & & & 28 & 29.947 & 3.160 \\
\hline Altura sinfisiana & 32 & $31.903 \mathrm{~b}$ & 3.516 & & & & & & & 28 & 32.606 & 3.110 \\
\hline Altura cuerpo mandibular & 32 & 19.690 & 4.544 & & & & & & & 28 & 19.353 & 4.608 \\
\hline Espesor cuerpo mandibular máximo & 32 & 16.316 & 1.668 & & & & & & & 28 & 15.921 & 1.630 \\
\hline Angulo mandibular (Gonion) & 30 & 123.194 & 7.253 & & & & & & & 27 & 122.379 & 6.758 \\
\hline
\end{tabular}

Tabla 2. Deformacion artificial. Valores estadísticos y pruebas de distribución normal. N: número de observaciones; MD: media; S: desviación estándar; a y b rechazo de la hipótesis nula de asimetría a nivel de 0.05 y 0.01 de probabilidad; c y d rechazo de la hipótesis nula de curtosis a nivel de 0.05 y 0.01 de probabilidad. 


\begin{tabular}{|c|c|c|c|}
\hline \multirow{2}{*}{ Variables } & 1 & 2 & \\
\hline & M' & gl & $\mathbf{F}$ \\
\hline Longitud máxima (glabela) & $9.601 *$ & 153 & $28.3313 * *$ \\
\hline Ancho máximo & 0.603 & 154 & $18.0725 * *$ \\
\hline Altura basilobregmática & 2.210 & 152 & $2.9107 *$ \\
\hline Altura poriobregmática & 0.451 & 152 & $3.0156^{*}$ \\
\hline Diámetro frontal mínimo & $8.391 *$ & 154 & $6.2223 * *$ \\
\hline Diámetro frontal máximo & 4.355 & 153 & $11.4126^{* *}$ \\
\hline Ancho bimastoideo & 3.635 & 154 & $3.5753 *$ \\
\hline Ancho bimastoideo máximo & 2.739 & 154 & $7.9639 * *$ \\
\hline Ancho biauricular & 4.382 & 154 & $3.7990 *$ \\
\hline Diámetro nasobasilar & 3.129 & 151 & 0.9123 \\
\hline Diámetro alveolobasilar & 4.821 & 151 & 1.5803 \\
\hline Longitud del forámen magnum & 1.754 & 149 & 1.4165 \\
\hline Ancho del forámen magnum & 4.050 & 150 & 1.8378 \\
\hline Ancho frontomalar & 3.173 & 154 & 1.8446 \\
\hline Ancho biastérico & 1.077 & 154 & $3.7988 *$ \\
\hline Diámetro nasion-sphenobasion & 2.523 & 154 & 0.6038 \\
\hline Diámetro alvéolo-sphenobasion & 4.525 & 153 & 1.5686 \\
\hline \multicolumn{4}{|l|}{ Diámetro basion-gnation } \\
\hline Curva transversal & 1.797 & 153 & $5.0536 * *$ \\
\hline Curva horizontal por opistocráneo & 0.508 & 153 & $8.5432 * *$ \\
\hline Curva nasion-bregma & 8.467 & 154 & $3.3324 *$ \\
\hline Curva bregma-lambda & 3.655 & 154 & 0.3495 \\
\hline Curva lambda-opistion & 1.029 & 151 & $14.0775 * *$ \\
\hline Ancho bicigomático & 1.623 & 151 & 0.7497 \\
\hline Altura del pómulo & 4.597 & 153 & 1.4133 \\
\hline Altura nasoalveolar & 2.584 & 153 & 2.0382 \\
\hline Altura de la nariz & 2.910 & 153 & 0.4955 \\
\hline Ancho apertura pyriformis & 1.865 & 153 & 1.5943 \\
\hline Distancia interorbitaria anterior & 5.058 & 154 & $2.7079 *$ \\
\hline Ancho biorbitaria & 2.090 & 153 & $2.7925 *$ \\
\hline Ancho de la órbita & 3.885 & 154 & $3.3656 *$ \\
\hline Altura de la órbita & 0.363 & 154 & 0.3719 \\
\hline Longitud del paladar & 2.671 & 151 & 0.4371 \\
\hline Ancho del paladar & 1.499 & 153 & $3.6080 *$ \\
\hline Altura orbitoalveolar & 4.888 & 145 & 0.2052 \\
\hline Ancho bimaxilar máximo & 1.935 & 152 & 0.0720 \\
\hline \multicolumn{4}{|l|}{ Altura nasion-gnation } \\
\hline Ancho maxiloalveolar & 3.130 & 144 & 1.2528 \\
\hline Longitud maxiloalveolar & 2.074 & 146 & 0.3556 \\
\hline \multicolumn{4}{|l|}{ Ancho bicondíleo } \\
\hline \multicolumn{4}{|l|}{ Ancho bigoníaco } \\
\hline \multicolumn{4}{|l|}{ Longitud de la mandíbula } \\
\hline \multicolumn{4}{|l|}{ Altura rama montante } \\
\hline \multicolumn{4}{|l|}{ Altura rama montante mínima } \\
\hline \multicolumn{4}{|l|}{ Ancho rama montante } \\
\hline \multicolumn{4}{|l|}{ Ancho rama montante mínimo } \\
\hline \multicolumn{4}{|l|}{ Altura sinfisiana } \\
\hline \multicolumn{4}{|l|}{ Altura cuerpo mandibular máximo } \\
\hline \multicolumn{4}{|l|}{ Espesor cuerpo mandibular } \\
\hline Angulo mandibular (Gonion) & & & \\
\hline
\end{tabular}

Tabla 3. Deformacion artificial. Dócimas de homogeneidad entre varianzas y de diferencia entre promedios. $1=$ Homogeneidad entre varianzas; 2 = Efecto deformación; $*$ rechazo de la hipótesis nula a nivel de 0.05 de probabilidad; *** rechazo de la hipótesis nula a nivel de 0.01 de probabilidad. 
Con respecto a las pruebas de distribución, se observan varios casos de rechazo de la hipótesis de normalidad. Esto ocurre fundamentalmente entre los ejemplares tabulares erectos, a nivel del $1 \%$ de probabilidad, para las variables: diámetro frontal máximo (curtosis), curva nasion bregma (asimetría) y altura nasio alveolar (asimetría y curtosis). Entre los circulares erectos se presentan problemas de curtosis y en oblicuos un caso de curtosis y otro de simetría distinta de la normal. En los ejemplares normales, la curva lambda opistion presenta en forma recurrente una distribución que se aparta de la normalidad. La prueba de homocedasticidad dio como resultado dos rechazos al 5\% de probabilidad para la longitud máxima del cráneo y para el diámetro frontal mínimo.

Los efectos de los distintos tipos deformatorios, con independencia del sexo y de la edad de los cráneos, se pusieron de manifiesto en un conjunto de variables que incluye: la longitud, ancho y alturas de la bóveda, los diámetros frontales, los anchos de la base craneal, las curvas transversal y horizontal y los segmentos frontal y occipital del perfil sagital. En el esplacnocráneo existe una incidencia menor que se manifiesta a nivel de la distancia interorbitaria anterior, del ancho de la órbita y biorbitaria y del ancho del paladar. Para el resto de las variables (35) no hemos obtenido pruebas suficientes para rechazar la hipótesis nula de igualdad entre los valores medios.

Los resultados expuestos deben ser evaluados de acuerdo con el cumplimiento de los supuestos que condicionan el análisis de la varianza, cuales son el de distribución normal y el de igualdad entre varianzas. Sugerimos, por la experiencia adquirida, la aceptación de las dócimas de diferencia entre medias cuando el rechazo de la hipótesis nula se realiza a dos niveles, aun cuando alguno de tales supuestos no se cumpla. Es necesario advertir que esta experiencia aporta información global sobre el influjo de la práctica cultural en la morfogénesis craneana y no en particular sobre un determinado tipo deformatorio. Por otra parte, sus resultados son válidos si aceptamos que no existe variación geográfica ni cronológica involucrada, es decir, si se supone que los ejemplares que integran la muestra son de origen local y contemporáneos.

En líneas generales, el comportamiento de las variables métricas afectadas por la deformación artificial parece ser consistente dentro de cada tipo con respecto a los valores normales. Los ejemplares tabulares erectos presentan una mayor proporción de distribuciones no normales, lo cual puede estar indicando también el efecto de la práctica cultural. Esta modalidad se expresa en Coyo Oriente a través de una disminución en el desarrollo anteroposterior del cráneo y en su expansión lateral, del aplastamiento del frontal a nivel de su diámetro máximo y de la restricción del crecimiento longitudinal de este hueso y del occipital, así como del mayor desarrollo de las curvas horizontal y transversal.

Con respecto a las formas circulares también la tendencia general de los valores medios es bastante coherente, aunque los grados de libertad son escasos. En las formas erectas se produjo una restricción al crecimiento del perímetro horizontal reflejado correlativamente por la disminución de la longitud anteroposterior, de la anchura máxima de la bóveda y del diámetro frontal máximo, con un consecuente incremento en la altura del cráneo, en la curva transversal y en el segmento occipital de la sagital. Por su parte, la restricción al crecimiento lateral en las formas oblicuas parece haber sido compensada por un incremento de la longitud; los diámetros frontales indican una fuerte compresión, al igual que las curvas transversal y horizontal. Posiblemente los efectos observados en la región orbitaria en general se deban a esta práctica.

En Pisagua, donde existen todos los tipos deformatorios (tabular erecto y oblicuo, circular erecto y oblicuo), hemos encontrado un esquema muy similar en la respuesta de las variables métricas (Cocilovo, en prensa), a pesar de los diferentes diseños experimentales empleados. Los primeros resultados obtenidos en Morro de Arica demuestran el efecto global de la deformación circular (Cocilovo et al. 1982), que en esta muestra se encuentra representada por las formas oblicua y erecta. El influjo particular de cada una de ellas fue analizado posteriormente empleando perfiles sagitales (Mendonça et al. 1983, 1986), pero hasta la fecha no existe un estudio similar para las variables métricas, lo que impide realizar un estudio comparativo. En Morro de Arica la deformación circular se manifiesta por un acortamiento de la longitud al inion y del ancho máximo, una expansión a nivel del ancho biastérico y efectos menores en la porción esplácnica (Cocilovo et al. 1982), incrementos que no fueron observados en Pisagua ni en Coyo. 
En Trelew hemos logrado un mejor modelo sobre los efectos de la deformación tabular erecta (Cocilovo 1978) que, a nivel de la bóveda y del esplacnocráneo, coincide con lo observado en Coyo. En el análisis global de una muestra de San Pedro de Atacama (Cocilovo y Varela Ms) se logró una mayor aproximación al comparar ejemplares tabulares erectos y oblicuos con normales. En efecto, en ambas prácticas se verifica un acortamiento anteroposterior y una expansión del ancho de la bóveda, sin una respuesta en la altura. En la forma oblicua se incrementa la longitud máxima, el diámetro alveolobasilar, alveolo-sphenobasion y las alturas de la nariz y de la órbita; mientras que en la erecta se observa una expansión del frontal, a nivel de su diámetro máximo, y de la curva transversal.

Hasta aquí es toda la información disponible; falta todavía mucho trabajo para lograr una imagen más nítida sobre el efecto de las deformaciones artificiales en la morfogénesis craneana. Por ejemplo, las diferencias entre circulares erectos y oblicuos pueden ser analizadas más en detalle reclasificando la muestra de Morro de Arica. En Pisagua, San Pedro de Atacama y Coyo Oriente se debe realizar una dócima de hipótesis a posteriori para comparar los valores medios de cada tipo entre sí y con los ejemplares normales. Con esto, podrá obtenerse una información más apropiada que caracterice las diferentes prácticas culturales.

Por último, deseamos dejar planteado un problema clásico, cual es el de considerar a un determinado tipo deformatorio como marcador biocultural. A pesar de la información disponible en nuestro banco de datos, creemos que es necesario realizar una experiencia específica sobre el particular, pues constituye un tema más complejo que lo comúnmente aceptado. Así, no sería extraño que un mismo tipo deformatorio varíe en sentido espacial y temporal, trayendo como consecuencia la necesidad de elaborar definiciones más detalladas y precisas, susceptibles de ser probadas estadísticamente. En tal sentido, es suficientemente demostrativa la variabilidad presentada por Weiss $(1961,1962)$ en Perú por llautu (circulares) y por la cuna (tabulares) dentro de las dos grandes familias, o los diversos tipos reconocidos en Norteamérica por Neuman (1942). En Coyo aparentemente, por la existencia de ejemplares circulares y por el resto de antecedentes arqueológicos, se podría inferir un contacto altiplánico directo, sólo queda un paso para pensar en Tiwanaku. Pero este tipo de deformación se encuentra también presente en todos los cementerios de la costa norte de Chile, en Morro de Arica (3000 AC) y en Pisagua (300 AC-1450 DC), lo que involucra una cuota de duda razonable sobre aquella explicación, máxime cuando advertimos que carecemos de un registro moderno para el área altiplánica circuntiticaca.

En nuestro relevamiento hemos encontrado algunas discrepancias con trabajos anteriores referidos a San Pedro. Por ejemplo, Munizaga (1969) no registra los tipos circulares, en tanto que nosotros no hemos identificado para Coyo ejemplares tabulares oblicuos, los que según este autor predominan en la Fase San Pedro II. Nuestra serie presenta un predominio neto de tabulares erectos, tipo deformatorio que, de acuerdo con aquel trabajo, es más frecuente al comienzo y al final del desarrollo cultural de San Pedro de Atacama. El modelo de distribución propuesto por Munizaga (1969) fue confirmado, al menos para San Pedro III, con los datos del sector tardío de Quitor 6, sitio en el que Costa (1985) comprueba la existencia de formas asimilables a las tabulares erectas y oblicuas. Estas discrepancias tal vez sean más aparentes que reales, pero en todo caso sugieren la necesidad de realizar una revaluación moderna partiendo de definiciones más precisas de cada práctica cultural y de un análisis más completo de su distribución espacial $y$ temporal en el norte de Chile.

Agradecimientos Deseamos expresar nuestro sincero agradecimiento al Dr. Francisco Rotthammer por su constante y permanente estímulo. A la Dra. Amy Oakland por su apoyo para la realización del viaje de estudio. A la Prof. S. G. Valdano por su ayuda en el procesamiento de la información métrica. Este trabajo fue realizado con fondos de la Fundación Antorchas (Proyecto 11766/89) y convenio CONICET-CONICYT (Argentina y Chile, Resolución 1405/90). 


\section{REFERENCIAS CITADAS}

BAFFI, E. I., Ms. Antropología física de la región valliserrana. III Relaciones biológicas con selvas occidentales. Primer Informe Beca Perfeccionamiento CONICET (1988), Buenos Aires.

BERENGUER, J. y P. DAUELSBERG, 1989. El Norte Grande en la órbita de Tiwanaku (400 a 1200 DC). En Culturas de Chile, Prehistoria. Desde sus orígenes hasta los albores de la Conquista, J. Hidalgo, V. Schiappacasse, H. Niemeyer, C. Aldunate e I. Solimano (Eds.), pp. 129-180. Editorial Andrés Bello, Santiago.

BLISS, C. I., 1967. Statistic in biology. McGraw-Hill Book, Nueva York.

BORDACH, M. A., 1985. La determinación de la edad en el esqueleto humano. Comechingonia.

1989. La determinación del sexo en el esqueleto humano. Serie Didáctica. Cuaderno 4.

COCILOVO, J. A., 1973. Dimorfismo sexual y deformación craneana artificial en patagones de Chubut. Actas del II Simposio Internacional de Ciencias Morfológicas, pp. 633642. Córdoba.

1975. Estudio de dos factores que influencian la morfología craneana en una colección andina: El sexo y la deformación artificial. Revista del Instituto de Antropología 2: 197-212.

1978. Estudio de dos factores que influyen en la morfología craneana en una población patagónica: El sexo y la deformación artificial. Arqhivos de Anatomía e Antropología 3 (3): 113-141.

_En prensa. Biología del grupo prehistórico de Pisagua, costa norte de Chile. Antropología Biológica.

COCILOVO, J. A. y E. I. BAFFI, 1985. Contribución al conocimiento de las características biológicas de la población prehistórica de Puerta de La Paya (Salta). Runa 15: 153-178.

COCILOVO, J. A. y H. H. VARELA, Ms. La deformación craneana artificial en la población prehistórica de San Pedro de Atacama.

COCILOVO, J. A., F. ROTTHAMMER, S. QUEVEDO y E. LLOP, 1982. Microevolución de poblaciones prehistóricas del área andina III. La población del Morro de Arica. Craneometría. Revista de la Universidad Nacional de Río Cuarto 2 (2): 91-111.

COCILOVO, J. A., M. V. ZAVATTIERI y M. A. COSTA, 1994. Biología del grupo prehistórico de Coyo Oriental (San Pedro de Atacama, norte de Chile): I Dimorfismo sexual y variación etaria. Estudios Atacameños 11, este volumen.

COMAS, J., 1966. Manual de antropología física. Universidad Autónoma de México, México D. F.

COSTA, M. A., 1985. Quitor 6: Sector tardío. Memoria para optar al Título de Arqueólogo. Departamento de Arqueología, Universidad del Norte, Antofagasta.
- 1988. Reconstrucción física y cultural de la población tardía del cementerio de Quitor 6 (San Pedro de Atacama). Estudios Atacameños 9: 99-126.

DAVID, H. A., O. HARTLEY y F. E. PEARSON, 1954. The distribution of the ratio in single normal sample of range to standard deviation. Biometrika 41 (3-4): 482-497.

DEMBO, A. y J. IMBELLONI, s/f. Deformaciones intencionales del cuerpo humano. Humanior Sec. A 3.

DI RIENZO, J. A. y J. A. COCILOVO, 1984. El análisis del material osteológico. Aplicación de una técnica objetiva de clasificación. Revista de la Universidad Nacional de Río Cuarto 4 (1): 57-74.

FALKENBURGER, F., 1938. Recherches anthropologiques sur la dèformation artificielle du crane. Journal de la Société des Américanistes 30 (1): 1-69.

GUICHON, R. A., Ms. Relaciones y afinidades biológicas de las poblaciones indígenas de la Patagonia Meridional. Informe Beca Formación Superior CONICET (1989), Buenos Aires.

HRDLJCKA, A., 1910. Artificial deformation of the human skull with special reference to America. Actas del XVII Congreso Internacional de Americanistas, pp. 147-149, Buenos Aires.

IMBELLONI, J., 1925. Deformaciones intencionales del cráneo en Sudamérica. Revista del Museo de La Plata 18: 329-407.

1932. América cuartel general de las deformaciones craneanas. Actas del XXV Congreso Internacional de Americanistas, La Plata, vol. 1, pp. 60-68. Buenos Aires.

1933. Los pueblos deformadores de los Andes. La deformación intencional de la cabeza como arte y como elemento diagnóstico de las culturas. Anales del Museo Argentino de Ciencias Naturales Bernardino Rivadavia 37: 209-254.

1950. Cephalic deformation of the indians in Argentine. Handbook of Southamerican Indians 6: 53-55, Smithsonian Institution, Washington D. C.

MENDONÇA, O. J. y J. A. DI RIENZO. La deformación craneana artificial 1981-82 de la serie masculina de Morro de Arica (Chile), Segunda Parte. Revista de la Sociedad Argentina de Antropología 14 (2): 49-66.

MENDONÇA, O. J., M. A. BORDACH y J. A. DI RIENZO, 1986. La deformación craneana artificial en la serie femenina de Morro de Arica (Chile), Primera Parte. Runa 16: $85-102$

MENDONÇA, O. J., J. A. DI RIENZO y M. A. BORDACH, 1983. La deformación craneana artificial en la serie masculina de Morro de Arica (Chile), Primera Parte. Revista de la Universidad Nacional de Río Cuarto 3 (1): 27-40.

MCNEILL, R. W. y G. N. NEWTON, 1965. Cranial base morphology in association with intentional cranial vault deformation. American Journal of Physical Anthropology 23: 241-254. 
MOSS, M. L., 1958. The pathogenesis of artificial cranial deformation. American Journal of Physical Anthropology 16 (3): 269-286.

MUNIZAGA, J., 1969. Deformación craneana intencional en San Pedro de Atacama. Actas del V Congreso Nacional de Arqueología Chilena, pp. 129-134. La Serena.

NEUMAN, G. K., 1942. Types of artificially cranial deformation in the eastern United States. American Antiquity 7: 306-310.

OETTEKING, B., 1922. On mophological changes in artificially deformed skull from the North Pacific coast. Anales XX Congreso Internacional de Americanistas, vol 2, pp. 25-35.

OSSENBERG, N. S., 1970. The influence of artificial cranial deformation on discontinuous morphological traits. American Journal of Physical Antropology 33 (3): 357-372.

PUCCIARELLI, H. M., 1972. Relaciones entre huesos wormianos y otros rasgos neurocraneanos sobre un grupo racial homogéneo. Anales de la Sociedad Científica Argentina 194 (5-6): 233-243.

1973a. Experimental technique for cranial deformation in growing rats. Acta Phys. Latino Americana 23: 141-147.
_ 1973b. Comparative and experimental craniology on adaptative traits. Actas del II Simposio Internacional de Ciencias Morfológicas, pp. 617-621.

- 1974. The influence of experimental deformation on neurocranial wormian bones in rats. American Journal of Physical Anthropology 41 (1): 29-38.

RAO, C. R., 1952. Advanced statistical methods in biometrics research. Ed. John Wiley \& Sons, Nueva York.

SHAPIRO, S. S. y M. B. WILK, 1965. An analysis test for normality (complete sample). Biometrika 52 (3-4): 591-611.

VARELA, H. H., J. A. COCILOVO y M. A. COSTA, 1990. El dimorfismo sexual en la población prehistórica de San Pedro de Atacama. Chungara.

WILDER, H., 1920. Laboratory mannual of anthropometry. P. Blakiston's Son and Co., Filadelfia.

WEISS, P., 1961. Osteología cultural. Prácticas cefálicas. $2^{\text {da. }}$ Parte. Lima.

1962. Tipología de las deformaciones cefálicas, Revista del Museo Nacional 31: 15-42. 